\title{
Mathematics in Computer Science: After 10 Years
}

\author{
Ilias S. Kotsireas
}

Published online: 24 March 2017

(C) Springer International Publishing 2017

\section{Introduction}

The first decade of uninterrupted publication of Mathematics in Computer Science (MCS) has elapsed. MCS published ten volumes from 2007 to 2016, each volume usually having four issues, either special or regular. Google Scholar shows that there are more than 2200 citations of MCS papers. The dblp online database indexed all papers and the AMS MathSciNet online database indexed close to 500 papers and forewords to special issues and special foci published in MCS. There are about 700 researchers who have authored MCS papers.

The milestone of the first decade of MCS now comes to existence, due to the contributions of many individuals:

- researchers from the community who submitted papers for publication in MCS,

- referees who provided valuable feedback to authors of submitted papers,

- members of the MCS Editorial Board who made their expertise available for fruitful interactions between authors and referees,

- guest editors of special issues and special foci who assembled excellent collections of papers on common themes, and

- MCS Editors-in-Chief, Dongming Wang and Zhiming Zheng, whose determination and hard work continued on, from day one and without interruption, for one decade.

I like to mention that Dongming also served as MCS Managing Editor for the past 10 years. He deserves special recognition for his dedication of service and tireless effort.

MCS is indexed in a number of bibliographic databases, including Mathematical Reviews, SCOPUS, Zentralblatt Math, MathSciNet, Google Scholar, Academic OneFile, DBLP, Expanded Academic, MathEDUC, OCLC, SCImago, and Summon by ProQuest.

\section{Papers by Distinguished Authors}

MCS features a tradition of publishing high-quality research papers at the intersection of Mathematics and Computer Science and I would like to provide a few excerpts, reproduced with comments, from some of the MCS papers by extremely high-profiled scientists.

I. S. Kotsireas $(\varangle)$

Wilfrid Laurier University, Waterloo, ON, Canada

e-mail: ikotsire@wlu.ca 
- Alan Bundy, 2007 Herbrand Award Winner and Fellow of the Royal Society, from the University of Edinburgh. In their MCS paper [5], Bundy and his co-authors Daniel Raggi, Gudmund Grov, and Alison Pease argue that representation determines how we can reason about a specific problem. Sometimes one representation helps us to find a proof more easily than others. Most current automated reasoning tools focus on reasoning within one representation. There is, therefore, a need for the development of better tools to mechanise and automate formal and logically sound changes of representation. They look at examples of representational transformations in discrete mathematics, and show how they have used tools from Isabelles Transfer package to automate the use of these transformations in proofs. They give an overview of a general theory of transformations that they consider appropriate for thinking about the matter, and they explain how it relates to the Transfer package. They show a few reasoning tactics they developed in Isabelle to improve the use of transformations, including the automation of search in the space of representations. They present and analyse some results of the use of these tactics.

- Yuri I. Manin, Former Director (1995-2005) of Max-Planck-Institut für Mathematik.

In their first MCS paper [3], Manin and Marcolli start from the obeservation that the theory of error-correcting codes is concerned with constructing codes that optimize simultaneously transmission rate and relative minimum distance. These conflicting requirements determine an asymptotic bound, which is a continuous curve in the space of parameters. The main goal of the paper is to relate the asymptotic bound to phase diagrams of quantum statistical mechanical systems. They first identify the code parameters with Hausdorff and von Neumann dimensions, by considering fractals consisting of infinite sequences of code words. They then construct operator algebras associated to individual codes. These are Toeplitz algebras with a time evolution for which the KMS state at critical temperature gives the Hausdorff measure on the corresponding fractal. They extend this construction to algebras associated to limit points of codes, with non-uniform multi-fractal measures, and to tensor products over varying parameters.

In their second MCS paper [4], Manin and Marcolli start from the observation that any natural language can be considered as a tool for producing large databases (consisting of texts, written, or discursive). This tool for its description in turn requires other large databases (dictionaries, grammars. etc.). Nowadays, the notion of database is associated with computer processing and computer memory. However, a natural language resides also in human brains and functions in human communication, from interpersonal to intergenerational one. They discuss in the survey/research paper mathematical, in particular geometric, constructions, which help to bridge these two worlds. In particular, they consider the Vector Space Model of semantics based on frequency matrices, as used in Natural Language Processing. They investigate underlying geometries, formulated in terms of Grassmannians, projective spaces, and flag varieties. They formulate the relation between vector space models and semantic spaces based on semic axes in terms of projectability of subvarieties in Grassmannians and projective spaces. They interpret Latent Semantics as a geometric flow on Grassmannians. They also discuss how to formulate Gärdenfors notion of "meeting of minds" in their geometric setting.

- Bjarne Stroustrup, Creator of the C++ Programming Language, from Texas A\&M University.

In the MCS paper [1], Stroustrup and his co-author Gabriel Dos Reis present a systematic representation of $\mathrm{C}++$, called IPR, for complete semantic analysis and semantics-based program transformations. They describe the ideas and design principles that shaped the IPR. In particular, they describe how general type-based unification is key to minimal compact representation, fast type-safe traversal, and scalability. For example, the representation of a fairly typical non-trivial C++ program in GCC 3.4.2 was 32 times larger than its IPR representation; this led to significant improvements to GCC. IPR is general enough to handle real-world programs involving many translation units, archaic programming styles, and generic programming using $\mathrm{C}++0 \mathrm{x}$ extensions that affect the type system. The difficult issue of how to represent irregular ( $\mathrm{ad} \mathrm{hoc}$ ) features in a systematic (non-ad hoc) manner is among the key contributions of the paper. The IPR data structure can represent all of $\mathrm{C}++$ with just 157 simple node types; to compare the ISO C++ grammar has over 700 productions. The IPR is used for a variety of program analysis and transformation tasks, such as visualization, loop simplification, and concept extraction. Finally, they report impacts of their work on existing C++ compilers.

- Lloyd N. Trefethen, Former President of SIAM and Fellow of the Royal Society, from Oxford University. 
In his MCS paper [6], Trefethen argues that symbolic computation with functions of a real variable suffers from combinatorial explosion of memory and computation time. The alternative chebfun system for such computations is described, based on Chebyshev expansions and barycentric interpolation. The paper carries a dedication to Richard Brent on his 60th birthday.

- Shing-Tung Yau, 1982 Fields Medalist, from Harvard University (the namesake of Calabi-Yau manifolds, together with Eugenio Calabi).

In their MCS paper [2], Yau and his co-authors David Xianfeng Gu and Feng Luo discuss the discipline of computational conformal geometry, an inter-disciplinary field between mathematics and computer science. The work introduces the fundamentals of computational conformal geometry, including theoretical foundation, computational algorithms, and engineering applications. Two computational methodologies are emphasized: one is the holomorphic differentials based on Riemann surface theory and the other is surface Ricci flow from geometric analysis.

\section{Editorial Board}

January 2016 was a sad month for MCS, since Mirka Miller, member of the MCS Editorial Board, from the University of Newcastle in New South Wales, Australia, passed away suddenly (following a short illness). During her career Mirka held positions at the Universities of New England, Newcastle and Ballarat and conjoint positions at King's College, London and University of West Bohemia, Pilsen. She is fondly remembered by her many colleagues, collaborators, and students in computer science and mathematics. She was a prolific researcher; the AMS MathSciNet database lists more than 200 of her research papers. An MCS special issue dedicated to Mirka, with papers related to her research interests, is currently being undertaken. The special issue is entitled "Mathematical Models for Security Applications" and it will contain survey papers related (but not restricted) to the courses lectured at the CIMPA Research School with the same title, which was held in Havana, Cuba, from August 29 to September 9, 2016 and dedicated to the memory of Mirka. The guest editors of the special issue are Luis Ramiro Piñeiro (Universidad de la Havana), Josep M. Miret (Universidad de Lleida, Spain) and Edgar Martínez-Moro (Universidad de Valladolid, Spain).

In January 2017, I was appointed MCS Managing Editor for a 5-year term. In addition, the MCS Editorial Board has been expanded with six distinguished new members from Europe and the United States:

1. James H. Davenport, University of Bath, UK.

2. Ioannis Z. Emiris, University of Athens, Greece.

3. Laura Kovács, Technische Universität Wien, Austria.

4. Matilde Marcolli, Caltech, USA.

5. Edgar Martínez-Moro, Universidad de Valladolid, Spain.

6. Andrew Sommese, University of Notre Dame, USA.

On behalf of MCS, I would like to welcome the six new members to the Editorial Board very warmly and I am looking forward to working with them and other Editorial Board members closely to ensure the future success of MCS.

\section{Special Issues and Foci}

An important part of the success of MCS is the publication of special issues and special foci, guest-edited by the world's leading experts on selected research themes within the scope of MCS. Two of the overarching features of the special issues and special foci are that they contain state-of-the-art papers and that these papers underwent rigorous refereeing by experts. I would like to list the special issues and special foci published in the first decade of MCS and highlight two of them in the end of this section. The MCS website http://www.cc $4 \mathrm{~cm} .0 \mathrm{rg} / \mathrm{mcs}$ contains 
a continuously updated list of all past, ongoing, and future MCS special issues and special foci, with links to the calls for papers.

- Vol. 10, no. 3: Advances in Symbolic and Numeric Computation; Guest editors: Amélia Loja, José Alberto Rodrigues, and Ana C. Conceição.

- Vol. 10, no. 2: Graph Theory and Applications; Guest editors: Bharati Rajan, Ibrahim Venkat, and K. G. Subramanian.

- Vol. 10, no. 1: Geometric Computation; Guest editors: J. Rafael Sendra, Dongming Wang, and Jing Yang.

- Vol. 9, no. 2: Applications of Mathematics in Computer Engineering; Guest editors: Mirka Miller, Bharati Rajan, and Indra Rajasingh.

- Vol. 9, no. 1: Formal Proofs for Mathematics and Computer Science; Guest editors: Laurent Théry and Freek Wiedijk.

- Vol. 8, nos. 3-4: Interval Methods and Applications; Guest editors: Nacim Ramdani and Luc Jaulin.

- Vol. 8, no. 2: Computational Algebraic Geometry; Guest editors: Sandra Di Rocco and Josef Schicho.

- Vol. 8, no. 1: Enabling Domain Experts to Use Formalised Reasoning; Guest editors: Manfred Kerber, Christoph Lange, and Colin Rowat.

- Vol. 7, no. 4: Mathematics, Data and Knowledge; Guest editors: Xiaoyu Chen, Dongming Wang, and Xiangliang Zhang.

- Vol. 7, no. 1: Near Set Theory and Applications; Guest editors: Som Naimpally, Jim Peters, and Marcin Wolski.

- Vol. 6, no. 4: Constraint Solving and Complex Systems; Guest editors: Stefan Ratschan and Thomas Sturm.

- Vol. 6, no. 3: Mathematics and Algorithms for Computer-Aided Manufacturing, Engineering and Numerical Control; Guest editors: Rida Farouki, Hongbo Li, and Dingkang Wang.

- Vol. 6, no. 2: Matroids in Coding Theory and Related Topics; Guest editors: Ilias S. Kotsireas, Irene MrquezCorbella, and Edgar Martínez-Moro.

- Vol. 5, no. 4: Numerical Software Verification; Guest editors: Georgios Fainekos, Eric Goubault, Sylvie Putot, and Stefan Ratschan.

- Vol. 5, no. 3: Polynomial System Solving, System and Control, and Software Science; Guest editor: Hirokazu Anai.

- Vol. 5, no. 1: Graph Theory and Applications; Guest editors: Mirka Miller, Bharati Rajan, and Joe Ryan.

- Vol. 4, no. 4: Algorithms and Complexity at the Interface of Mathematics and Computer Science; Guest editors: Gert Vegter and Chee Yap.

- Vol. 4, nos. 2-3: Algebraic and Algorithmic Aspects of Differential and Integral Operators; Guest editors: Georg Regensburger, Markus Rosenkranz, and William Sit.

- Vol. 4, no. 1: Computational Geometry and Computer-Aided Geometric Design; Guest editors: Laureano Gonzalez-Vega and Sylvain Lazard.

- Vol. 3, no. 4: Advances in Combinatorial Algorithms II; Guest editors: Manolis Christodoulakis and Costas S. Iliopoulos.

- Vol. 3, no. 3: Authoring, Digitalization and Management of Mathematical Knowledge; Guest editors: Serge Autexier, Petr Sojka, and Masakazu Suzuki.

- Vol. 3, no. 2: Symbolic Computation and Cryptography; Guest editors: Jean-Charles Faugère and Ludovic Perret.

- Vol. 3, no. 1: Advances in Combinatorial Algorithms I; Guest editors: Mirka Miller and Koichi Wada.

- Vol. 2, no. 3: Symbolic Computation in Biology; Guest editors: Hirokazu Anai and Katsuhisa Horimoto.

- Vol. 2, no. 2: Management of Mathematical Knowledge; Guest editor: Manfred Kerber.

- Vol. 2, no. 1: Intensional Programming \& Semantics; Guest editors: Mehmet A. Orgun and John Plaice.

- Vol. 1, no. 4: Combinatorial Algorithms; Guest editors: Joseph Wun-Tat Chan and Maxime Crochemore.

- Vol. 1, no. 3: Modeling and Analysis of Complex Systems ; Guest editor: Ilias S. Kotsireas.

- Vol. 1, no. 2: Symbolic and Numeric Computation; Guest editors: Dongming Wang and Lihong Zhi.

- Vol. 1, no. 1: Algorithms and Complexity; Guest editors: Chee K. Yap and Hoon Hong. 
The inaugural special issue of MCS focused on algorithms for continuous problems and was edited by Chee K. Yap and Hoon Hong. In the foreword [9] they state that:

The study of computation spans a continuum, from the analysis of concrete algorithms (e.g., linear programming) to the general properties of complexity classes (e.g., polynomial-time recognizable problems). Both ends of this spectrum are highly developed areas of theoretical computer science, ranging from the field of algorithmics for concrete algorithms to the field of complexity theory for complexity classes. But both algorithmics and complexity theory are built largely on discrete algorithms. In contrast, a large part of mathematical computation is concerned with continuous algorithms. The development of "continuous algorithmics" and its associated complexity theory is an area that MCS hopes to nurture.

The special issue spanned more than 200 pages and contained 6 papers, by Lloyd N. Trefethen, Daniel Richardson, Sunayana Ghosh, Sylvain Petitjean, Gert Vegter, Vikram Sharma, Joris van der Hoeven, and Mohab Safey El Din. The MCS special issue on Algorithms and Complexity at the Interface of Discrete and Continuous Computations was edited by Gert Vegter and Chee K. Yap. In the foreword [7] they state that:

Theoretical algorithms and their complexity are central in foundational research at the interface between mathematics and computer science. There are many current challenges especially at the interface of continuous and discrete computation. For instance, how can we faithfully and efficiently discretize a continuous geometric object, or a continuous problem? Efficient new algorithmic techniques must be developed and analyzed. The inherent complexity of such problems needs to be characterized. Such considerations are central to current research areas such as (i) robust geometric algorithms, (ii) the surface-surface intersection (SSI) challenge in geometric modeling, (iii) mesh generation with topological guarantees, (iv) the development of a theory of real computation, and (v) the emerging field of numeric-algebraic computation. Such problems are the focus of this special issue.

This special issue spanned more than 200 pages, again, and contained 6 papers by Marc Mörig, Ivo Rässling, Stefan Schirra, Prashant Batra, Michael Sagraloff, Huaiping Yang, Bert Jüttler, Laureano Gonzalez-Vega, JeanDaniel Boissonnat, Arijit Ghosh, David Xianfeng Gu, Feng Luo, and Shing-Tung Yau.

\section{Future Directions and Prospects}

Moving forward to the second decade of its existence, MCS will look at expanding its scope to additional research directions and domains, such as Coding Theory, Discrete Mathematics, Automated Proofs, Constructive Combinatorics, Quantum Computing, Computational Geometry, Optimization and Data Science to name a few. All these areas encompass tools, techniques and methodologies that are very close to the MCS mandate. Therefore, promoting and strengthening the interactions between researchers from these areas and researchers from Computer Algebra and Symbolic Computation is a worthwhile endeavor, which will hopefully contribute in bridging the gap between them. To this effect, MCS will soon publish a special issue on "Algorithms and Big Data", edited by Costas Iliopoulos and Alessio Langiu (both from King's College London). In addition, a special issue with refereed selected papers presented at the ACA 2016 conference held in Kassel, Germany, August 1-4, 2016, will be focused on applications of computer algebra. This special issue will be edited by Thierry Dana-Picard (Jerusalem College of Technology), Wolfram Koepf (University of Kassel), Ilias Kotsireas (Wilfrid Laurier University), Zoltan Kovacs (RISC Linz), Alexander Prokopenya (Warsaw University of Life Sciences), and Werner Seiler (University of Kassel).

In their 2007 inaugural MCS editorial [8], Dongming Wang and Zhiming Zheng, MCS Editors-in-Chief, wrote that:

We hope that the endeavor with MCS will contribute to advancing mathematics and computer science and to enriching the scientific literature. 
I share their original vision of MCS and will strive to contribute to the future success of the journal, with the help of the MCS Editorial Board, guest editors of special issues, expert referees, and prospective authors.

Acknowledgements I am grateful to the dedicated Staff at Birkhäuser, Basel and the Production Editor at Springer Nature for their work and effort with MCS.

\section{References}

1. Dos Reis, G., Stroustrup, B.: A principled, complete, and efficient representation of C++. Math. Comput. Sci. 5(3), 335-356 (2011)

2. Gu, D.X., Luo, F., Yau, S.-T.: Fundamentals of computational conformal geometry. Math. Comput. Sci. 4(4), 389-429 (2010)

3. Manin, Y.I., Marcolli, M.: Error-correcting codes and phase transitions. Math. Comput. Sci. 5(2), 133-170 (2011)

4. Manin, Y.I., Marcolli, M.: Semantic spaces. Math. Comput. Sci. 10(4), 459-477 (2016)

5. Raggi, D., Bundy, A., Grov, G., Pease, A.: Automating change of representation for proofs in discrete mathematics. Math. Comput. Sci. 10(4), 429-457 (2016)

6. Trefethen, L.N.: Computing numerically with functions instead of numbers. Math. Comput. Sci. 1(1), 9-19 (2007)

7. Vegter, G., Yap, C.K.: Foreword. Math. Comput. Sci. 4(4), 385-387 (2010)

8. Wang, D., Zheng, Z.: Editorial. Math. Comput. Sci. 1(1), 1-2 (2007)

9. Yap, C.K., Hong, H.: Foreword. Math. Comput. Sci. 1(1), 3-7 (2007) 